\title{
АЛГОРИТМ СОКРАЩЕННОГО ВЫЧИСЛЕНИЯ ДИСКРЕТНОГО КОСИНУСНОГО ПРЕОБРАЗОВАНИЯ ФУРЬЕ
}

I. ARRO. DISKREETSE FOURIER' KOOSINUSTEISENDUSE KOONDARVUTUSALGORITM

1. ARRO. VERKURZTER ALGORITHMUS FUR DIE DISKRETE KOSINUS-FOURIER-TRANSFORMATION

$$
\text { (Представил Н. Алумяэ) }
$$

\section{1. Введение}

В практике цифровой обработки сигналов исходные величины являются вещественными. Образующиеся при этом массивы данных могут быть четными, нечетными или смешанными. Поэтому особое значение имеют алгоритмы, позволяющие высокоэффективно обрабатывать исходные данные непосредственно с учетом их особенностей.

Предлагается новый алгоритм косинусного преобразования Фурье вещественного массива данных, который выполняется вдвое меньшим количеством операций умножения и сложения (вычитания) по сравнению с количеством соответствующих операций алгоритма сокращенного вычисления дискретного преобразования Фурье $\left[{ }^{1,2}\right]$.

\section{2. Постановка задачи}

Требуется вычислить дискретное косинусное преобразование Фурье (ДКПФ) $X C(K)$ от исходного вещественного массива $Y(I), \quad I=$ $=\overline{0,(N-1)}, K=\overline{0, N / 2}(X C(K)=X C(N-K)), N=2^{L P}, L P$ - натуральное число, т. е.

$$
X C(K)=\sum_{I=0}^{N-1} Y(I) \cos (2 \pi I K / N)
$$

Выполняя преобразования

$$
C Z(I)=Y(I)+Y(N-I), \quad I=\overline{0, N / 2,} \quad(Y(N)=(0))
$$

и принимая за основу алгоритм сокращенного вычисления дискретного преобразования Фурье $\left[{ }^{1}\right]$, можно формулу (1) представить в виде композиции следующих последовательных независящих друг от друга выражений:

$$
\begin{aligned}
& X C(R(2 k+1))=C_{M}(R(2 k+1))+C_{M / 2}(R(2 k+1))+ \\
& +\ldots+C_{8}(R(2 k+1))+Z C(0), \quad R \leqslant N / 8, \\
& R=2^{r}, \quad r=\overline{0,(L P-3),} \quad k=\overline{0,(N / 4 R-1),}
\end{aligned}
$$




$$
\begin{aligned}
& C_{M / P}((2 k+1) N / M)=\sum_{I=0}^{M / 8 P-1} Z C((2 I+1) P) \times \\
& \quad \times \cos (2 \pi(2 I+1)(2 k+1) /(M / P)), \\
& P=2^{\alpha}, \quad M=2^{L M}, \quad L M=L P-r, \quad \alpha=\overline{0,(L M-3),} \\
& k=0,(M / 8 P-1), \\
& Z C(I)=C Z(I)-C Z(M / 2-I), \\
& C Z(I)=C Z(I)+C Z(M / 2-I), \\
& I=\overline{0,(M / 4-1),} \quad M=N / R .
\end{aligned}
$$

Таким образом, ДКПФ сведено к вычислениям нечетных косинусных трансформантов согласно формуле (4).

\section{3. Сокращенное вычисление нечетного косипусного трансформанта с периодом $N$}

Без ущерба для общности, далее, положим $M=N, P=I$ и представим $C_{N}(2 K+1)(K=\overline{0,(N / 8-1)}$ в следующем виде:

$$
\begin{aligned}
& C_{N}(2 K+1)=\sum_{q=0}^{N / 4 G-1} R C(Q ; 2 K+1), \\
& Q=4 q+1, \quad q=\overline{0,(N / 4 G-1),} \quad K=\overline{0,(G / 4-1)}, \\
& R C(Q ; 2 K+1)=\cos (2 \pi Q(2 K+1) / N) \cdot X C_{G}^{Q}(2 K+1)- \\
& -\sin (2 \pi Q(2 K+1) / N) X S_{G}^{Q}(2 K+1), \\
& X C_{G}^{Q}(2 K+1)=Z C(Q)+\sum_{I=1}^{G / 4-1}(Z C(N I / G+Q)+Z C(N I / G-Q)) \times \\
& \times \cos (2 \pi(2 K+1) I / G), \\
& X S_{G}^{Q}(2 K+1)=Z C(N / 4-Q)+\sum_{I=1}^{G / 4-1}(Z C(N I / G+Q)- \\
& -Z C(N I / G-Q)) \sin (2 \pi(2 K+1) I / G), \\
& 4 \leqslant G \leqslant N / 4 \text {. }
\end{aligned}
$$

Аналогично [ ${ }^{1}$ ] осуществим распространение области спектра $K=$ $=\overline{0,(G / 4-1)}$ в область $K=\overline{0,(N / 8-1)}$ путем замены $2 K+1$ на $G H \pm(2 K+1)$.

Тогда

$$
\begin{aligned}
& X C_{G}^{Q}(G H \pm(2 K+1))=X C_{G}^{Q}(2 K+1) \\
& X S_{G}^{Q}(G H \pm(2 K+1))= \pm X S_{F_{F}}^{Q}(2 K+1)
\end{aligned}
$$

и

$$
\begin{gathered}
R C(Q ; G H \pm(2 K+1))=\cos (2 \pi Q H /(N / G)) R C(Q ; 2 K+1) \mp \\
\quad=\sin (2 \pi Q H /(N / G)) R S(Q ; 2 K+1) \\
\begin{aligned}
R S(Q ; 2 K+1) & =\sin (2 \pi Q(2 K+1) / N) X C_{G}^{Q}(2 K+1)+ \\
& +\cos (2 \pi Q(2 K+1) / N) X S_{G}^{Q}(2 K+1) \\
H & =0, \overline{N / 4 G}
\end{aligned}
\end{gathered}
$$




$$
C_{N}(G H \pm(2 K+1))=\sum_{q=0}^{N / 4 G-1} R C(4 q+1 ; G H \pm(2 K+1)) .
$$

Здесь уместно указать, что нечетное синусное преобразование (формула (10))

$$
\left.X S(2 K+1)=\sum_{I=1}^{G / 4-1} Z S(I) \sin (2 \pi(2 K+1) H / G)\right)
$$

целесообразно выполнить через косинусное, так как при замене $I$ на $G / 4-I$ получим

$$
\begin{gathered}
\left.X S(2 K+1)=(-1) K \sum_{I=1}^{G / 4-1} Z S(G / 4-I) \cos (2 \pi(2 K+1) I / G)\right), \\
K=\overline{0,(G / 4-1) .}
\end{gathered}
$$

Таким образом, вычисление нечетиого косинусного трансформанта с периодом $N$ для любого $4 \leqslant G \leqslant N / 4$ выполнимо (формулы (7)-(17)) через нечетные косинусные преобразования с периодом $G$ и спектральное расширение (формулы (13) и (15)).

\section{4. Заключение}

Предложен алгоритм сокращенного вычисления дискретного косинусного преобразования Фурье (СДКПФ), который выполняется с наименьшим числом арифметических операций (по сравнению со всеми известными аналогичными процедурами). Алгоритм СДКПФ является рекурсивным и, следовательно, эффективно программируемым. На уровне четной части реализации $(C Z(I)$, формула (2)) дважды последовательное применение СДКПФ восстанавливает исходный массив. Это означает, что все преобразования спектрально-корреляционного анализа, которые выполнимы по прямому и обратному дискретным преобразованиям Фирье (ДПФ), могут быть реализованы на основе СДКПФ и сумма-разностных преобразований, необходимых для декомпозиции (или композиции) функции вещественного переменного на четные и нечетные части.

Так, ДПФ вещественного массива может быть выполнено, применяя в отдельности СДКПФ для четной и нечетной (с учетом формулы (17)) частей исходной реализации. Обратное ДПФ выполняется с помощью восстановления четной и нечетной частей и их композицией.

Особую роль СДКПФ играет в задачах вычисления свертки, поскольку теперь общее количество операций может быть поставлено в непосредственную зависимость от характера исходной реализации и весовой функции: обе функции могут быть только четными или нечетными, или содержать как четную, так и нечетную части.

\section{Л И ТЕ Р А Т У Р А}

1. Арро И. Изв. АН ЭССР. Физ. Матем., 36, № 1, 21-29 (1987).

2. Арро И. О. В кн.: Методы и средства измерения, преобразования и обработки информации. Таллин, АН ЭССР, 1987, 8-18.

Институт кибернетики Академии наук Эстонской ССР
Поступила в редакцию $22 / \mathrm{V} 1987$ 